Terapia Psicológica, vol. 39, no. 2 (julio 202I)

\begin{abstract}
do
\section{Variables psicológicas asociadas al estrés Postraumático en desmovilizados de las Fuerzas PSICOLÓGICA Armadas Revolucionarias de Colombia (FARC-EP)}

ISSN: 0718-4808

Psychological variables associated with post-traumatic stress in demobilized members of the Revolutionary Armed Forces of Colombia (FARC-EP)

\section{Stefano Vinaccia}

(iD) 0000-0001-5169-0871

stefanovinaccieunisinu.edu.co

Andrea Ortega

(iD) 0000-0003-0527-3377

a.ortega.bechara@unisinu.edu.co

1 Universidad del Sinú, Facultad de Ciencias de la Salud. Montería, Colombia.

Resumen:

Objetivo: evaluar la relación entre el estrés post traumático y variables psicológicas asociadas, en guerrilleros desmovilizados las Fuerzas Armadas Revolucionarias de Colombia (FARC-EP) en el departamento de Córdoba, Colombia. Método: estudio cuantitativo, transversal, de alcance correlacional. Participaron 48 guerrilleros mayores de edad (34 hombres y 14 mujeres) pertenecientes al Frente 58 de esta agrupación, a quienes se les aplicó los cuestionarios Lista de chequeo de estrés post traumático (PCL-5), la escala de distrés psicológica de Kessler, el cuestionario de creencias básicas (CBI), la escala de la esperanza de Herth y la escala de bienestar compuesto (WBC). Resultados: los participantes no presentaban síntomas de estrés post traumático, tenían bajos niveles de estrés y de cambios en las creencias básicas, igualmente presentaban altos niveles de esperanza y de bienestar físico y psicológico. Para detectar posibles predictores del TEPT se desarrolló un análisis de regresión múltiple en el cual el $48 \%$ de la varianza total fue explicado por el distrés psicológico y las creencias básicas. Conclusión: se evidencia la necesidad de desarrollar estudios que profundicen en las características del estado psicológico de los ex guerrilleros de la FARC-EP, con el fin de aclarar las variables involucradas que favorecen los procesos de reintegración a la vida civil.

Palabras clave: estrés post traumático; distrés; esperanza; bienestar; guerrilleros; FARC-EP; Colombia.
\end{abstract}

\title{
Abstract:
}

Objective: to evaluate the relationship between post-traumatic stress and associated psychological variables in demobilized guerrillas from the Revolutionary Armed Forces of Colombia (FARC-EP) in the department of Córdoba, Colombia. Method: we carried out a quantitative, cross-sectional study of correlational scope, in which 48 guerrillas of legal age participated (34 men and 14 women) belonging to the 58th Front of this group. The Post Traumatic Stress Checklist (PCL-5), the Kessler psychological distress scale, the basic beliefs questionnaire $(\mathrm{CBI})$, the Herth hope scale and the composite well-being scale (WBC) were applied. Results: the participants did not present symptoms of post traumatic stress, they had low levels of stress and changes in basic beliefs, they also presented high levels of hope and physical and psychological well-being. To detect possible predictors of PTSD, a multiple regression analysis was developed in which $48 \%$ of the total variance was explained by psychological distress and basic beliefs. Conclusion: there is evidence of the need to develop studies that delve into the characteristics of the psychological state of former FARC-EP guerrillas, in order to clarify the variables involved that favor the processes of reintegration into civilian life.

Keywords: post-traumatic stress; distress; hope; well-being; ex-combatants; FARC-EP; Colombia 
Variables psicológicas asociadlas al estrés Postraumático en desmovilizados de las Fuerzas Armadas Revolucionarias de Colombia (FARC-EP)

\section{Introducción}

El conflicto armado colombiano se ha prolongado durante más de 50 años y ha dejado aproximadamente 220,000 muertos, 6 millones de desplazados y más de 27,000 secuestrados, lo que ha supuesto un enorme coste social y económico para el país, así como enormes costes personales para los afectados (Aguilera, 2017; Centro Nacional de Memoria Histórica [CNMH],2015; Medina, 2017; Restrepo \& Gagley, 2011; Ríos, 2016; Ugarizza \& Pabón, 2017). La desmovilización de las Fuerzas Armadas Revolucionarias de Colombia (FARC-EP) tras el acuerdo de paz de 2016, implicó el inicio un proceso político y el proceso de concentración de excombatientes en Espacios Territoriales de Capacitación y Reincorporación en diferentes zonas del territorio colombiano (Estrada, 2013).

La investigación psicológica sobre combatientes irregulares en Colombia ha sido escasa, aún más sobre guerrilleros de las FARC-EP. El principal ensayo sobre el tema lo ha desarrollado Ardila (2017), quien describió los factores históricos que llevaron al nacimiento de este grupo guerrillero, analizando su vida cotidiana, los procesos de identidad, la vida sexual, su vida en medio de la guerra, y las perspectivas de su reintegración a la vida civil. De igual manera, Valencia y Daza (2011), desarrollaron un ensayo semejante donde evaluaron los procesos vinculación en adultos, en menores de edad, y en mujeres, analizando las motivaciones para pertenecer a estas fuerzas irregulares. Este estudio halló semejanzas entre los motivos de vinculación a los grupos armados ilegales, en comparación con las Fuerzas Armadas.

La investigación empírica con excombatientes en Colombia se podría dividir en dos áreas de estudio: investigaciones con una mirada de tipo psicosocial e investigaciones de tipo neuropsicológico y psiquiátrico.

Dentro de la perspectiva psicosocial, Amar et al. (2011) evaluaron a 37 excombatientes desmovilizados FARC, identificando la existencia de relaciones estadísticamente significativas entre indicadores estructurales y funcionales de las redes personales con sus niveles de calidad de vida física y psicológica. Se observaron redes pequeñas, con altos niveles de insatisfacción frente al apoyo social, y reducidos niveles de calidad de vida. Se registraron diferencias significativas en la percepción general de apoyo de las redes y la obtención objetiva de ayuda, así como correlaciones entre los niveles de participación social en las redes y la vitalidad psicológica de los individuos.

En la misma perspectiva, Pineda et al. (2017), realizaron un análisis sobre los procesos de inclusión laboral de las personas desmovilizadas en la ciudad de Medellín y su área metropolitana, la cual concentra el mayor número de desmovilizados del país, con cerca de 5433 individuos que se han reintegrado a la sociedad entre los años 2006 a 2016 . El estudio halló diversas barreras sociales, culturales y económicas que limitan el acceso al empleo por 


\section{S. Vinaccia \& A. Ortega}

parte de los desmovilizados, entre ellas la estigmatización social, la baja oferta laboral, el desconocimiento de las habilidades del desmovilizado, y las presiones de grupos al margen de la ley que pueden ocasionar el retorno de estas personas a los grupos armados ilegales.

Por otra parte, en el área neuropsicológica y psiquiátrica, Tobón et al. (2015), estudiaron los correlatos neuronales del procesamiento emocional en 40 excombatientes de las FARC y 20 controles. Se midieron los niveles de empatía y las funciones ejecutivas de todos los participantes. Los resultados sugieren dos perfiles empáticos: un grupo de excombatientes con puntuaciones de empatía normales, y un grupo de excombatientes con puntuaciones bajas en al menos una subescala de empatía.

De igual manera, Tobón et al. (2016) diseñaron un estudio cuyo objetivo fue describir las características psiquiátricas, cognitivas y del procesamiento emocional de un grupo de excombatientes del conflicto armado colombiano. Evaluaron a 63 excombatientes y 22 controles en diferentes dominios como el coeficiente intelectual, la función ejecutiva, la empatía y el perfil psiquiátrico. En comparación con los controles, los excombatientes mostraron con mayor frecuencia trastorno de personalidad antisocial y trastorno disocial de la conducta. En el dominio cognitivo, en los excombatientes se evidenció un peor desempeño en la función ejecutiva y un pobre distrés personal en el componente empático con respecto al grupo control.

Con relación a la evaluación psicológica de estos combatientes a nivel de estrés post traumático Baldovino (2014), evaluó 190 sujetos desmovilizados de las FARC. El estudio reportó la existencia de Trastorno por Estrés Postraumático (TEPT) en el 37.4\% de los evaluados, y $12.1 \%$ en riesgo de presentar TEPT. Adicionalmente, $24.1 \%$ presentaban síntomas de estado dispositivo, $13.6 \%$ presentaba alteración del estado del ánimo, 47.1\% percibían indefensión, y $17.9 \%$ experimentaron miedo.

Sobre esta misma línea de trabajo, De la Espriella et al. (2010), desarrollaron un estudio para analizar si los excombatientes que han tenido exposición repetida a eventos estresantes podrían cumplir con los requisitos del trastorno de estrés postraumático (TEPT).

Finalmente, Aponte y Zapata (2018), en participantes que adelantaron su proceso de reincorporación entre el 2010 y el 2014, encontraron que en el 31\% de esa población (unas 8,370 personas, entre las que hay exguerrilleros y ex paramilitares), el conflicto les dejó estrés postraumático. Con relación a la salud mental, un $27.3 \%$ sufrían ansiedad, un $26.2 \%$ padecían trastorno explosivo intermitente del control de los impulsos, un $23 \%$ convivía con estados maniacos, un 17,1\% tenía depresión, el $13.8 \%$ sufría de juego patológico y un 10\% experimentaban psicosis. 
Por otro lado en diferentes áreas del mundo en que han existido conflictos internos de larga duración como en Colombia (véase en África la República Democrática del Congo y el Burundi), diferentes estudios sobre combatientes desmovilizados de diferentes grupos armados que combatieron por años las fuerzas gubernamentales encontraron que presentaban una historia de eventos traumáticos con síntomas muy extendidos de TEPT (Koebach et al, 2021). Al mismo tiempo, estos combatientes a menudo declararon haber percibido la comisión de actos violentos como algo emocionante y atractivo, una experiencia denominada agresión apetitiva (Nandi, et al 2020; Nandi et al, 2015)

Los análisis de estos estudios pueden evidenciar que hay un vacío significativo a nivel de estudios de salud mental sobre excombatientes de las guerrillas colombianas, donde prácticamente se han centrado en el cómo la exposición a situaciones o eventos estresantes podía conformar un factor de riesgo para contraer determinadas enfermedades mentales dejando de lado el análisis de las características positivas de la vida psicológica de estas personas que les hubieran mantenido orientados hacia un polo saludable de salud durante todos los años que estuvieron al margen de la ley en lugares aislados centrados en actividades que podían en riesgo su vida y la vida de las personas que vivían en su entorno. Esto nos llevó a diseñar un estudio que pretendió evaluar las Relaciones entre estrés post traumático con variables salutogénicas (esperanza y bienestar), patogénicas (distrés psicológico) e intermediarias como creencias básicas en un frente de guerrilleros desmovilizados de las FARC-EP en Tierralta, Córdoba, en sus propios campamentos de concentración.

La hipótesis del estudio planteó que en los ex combatientes de la FARC los niveles de distrés psicológico serian el mejor predictor de la gravedad de los de los síntomas de estrés post traumático mientras los niveles de esperanza y bienestar tendrían un efecto de soporte positivo frente a los síntomas del estrés post traumático

\section{Método}

\section{Diseño}

La presente es una investigación cuantitativa, de alcance correlacional, con diseño transversal (Hernández, et al., 2014).

\section{Participantes}

Mediante un muestreo no probabilístico, fueron contactados 48 adultos exguerrilleros (34 hombres y 14 mujeres) desmovilizados del Frente 58 de las FARC-EP, quienes diligenciaron el 
el consentimiento informado para participar en la investigación.

\section{Instrumentos}

El cuestionario de Creencias Básicas "Core Beliefs Inventory" (CBI), elaborado por Cann et al. (2008), es una escala auto administrada que consta de 9 ítems que se puntúan en una escala Likert de 0 a 5 desde "nada" (0) a "gran grado" (5). La escala evalúa temas religiosos, creencias espirituales, fortalezas y debilidades personales/interpersonales, y significado de la vida. El CBI presentó un índice de fiabilidad de alfa de Cronbach de .87 .

La escala de la Esperanza de Herth "The Herth Hope Scale" (HHS), desarrollada por Herth (1991), es una escala autoadministrada de 12 ítems con una puntuación total máxima de 40 puntos, en la que una mayor puntuación indica mayor nivel de esperanza. La escala presentó un índice de fiabilidad de alfa de Cronbach de .77 .

La Lista de Chequeo de Estrés Post Traumático para el DSM-5 (PCL-5), desarrollada por Weathers et al. (2013), es una medida de autoinforme de 20 ítems que evalúa los síntomas del DSM-V asociados al PTSD. La escala de calificación tipo Likert de autoinforme es $0-4$ para cada síntoma. La escala presentó un índice de fiabilidad de alfa de Cronbach de .87 .

La Kessler Psychological Distréss Scale (K10), elaborada por Kessler et al. (2002), es una medida de autoinforme de 10 ítems destinada a producir una medida global de angustia basada en preguntas sobre los niveles de ansiedad y los síntomas depresivos que una persona ha experimentado en el período más reciente de 4 semanas. Las opciones de respuesta se basan en una escala tipo Likert de 5 puntos que va de 1 (ninguna de las veces) a 5 (todo el tiempo). Las respuestas se suman para crear un puntaje total (rango $=10-50)$ con puntajes más altos que significan más angustia psicológica. La escala presentó un índice de fiabilidad de alfa de Cronbach de .92

La escala Well-Being Composite (WBC), desarrollada por Greenaway et al. (2015), es una escala autoadministrada de 3 ítems. El primer ítem mide el grado de satisfacción con la vida actual calificado en una escala que varía de 1 (completamente insatisfecho) hasta 10 (completamente satisfecho). El segundo ítem mide felicidad en una escala que va de 1 (muy feliz) a 4 (muy poco feliz). Y el tercer ítem su estado de salud actual que va de 1 (muy bueno) a 4 (muy pobre). 


\section{Procedimiento}

Antes de iniciar la fase de campo de la investigación que implicaba la aplicación de las pruebas psicométricas se llevó a cabo la traducción y la retro traducción de los instrumentos por parte de traductores oficiales. Posteriormente se llevó a cabo el proceso de adaptación cultural de los cuestionarios, según los criterios de Alexandre y Guirardello (2002). El trabajo de adaptación cultural con los cuestionarios CBI, HHS, PCL-5, K-10 y WBC consistió en la aplicación de las pruebas a un grupo de 10 personas delmismo contexto cultural regional donde se iba a desarrollar la investigación; luego de responder los cuestionarios fueron entrevistados para identificar palabras o preguntas de difícil comprensión, evaluar la aceptabilidad y hacer comentarios sobre estos de una forma general, registrándose además el tiempo necesario para completarlo y la comprensión respectiva en el sistema de respuesta de cada ítem de los cuestionarios. No se presentaron dificultades que ameritaran cambios, dado que las personas reportaron entendimiento y comprensión de los ítems en todos los instrumentos psicométricos evaluados.

Posteriormente la investigación fue aprobada por la junta de revisión institucional (junta de ética) de la Universidad del Sinú. El equipo de investigadores viajo al corregimiento de Gallo, donde estaban situados el territorio de capacitación y reincorporación los guerrilleros desmovilizados del Frente 58 de las FARC-EP, donde fueron aplicados los instrumentos de evaluación. El proceso de levantamiento de datos duró aproximadamente 40 minutos. Previamente se les había explicado los objetivos de la investigación y luego de la firma del consentimiento informado por parte de cada participante se diligenciaron las pruebas de manera individual.

\section{Análisis de datos}

Mediante la prueba de normalidad Shapiro-Wilk fue posible establecer que los puntajes del $\mathrm{CBI}, \mathrm{HHS}, \mathrm{PCL}-5, \mathrm{~K} 10$ y WBC presentaban una distribución normal en la muestra del estudio, lo que permitió después de la evaluación de los datos sociodemográficos, analizar las medidas de tendencia central de todos los instrumentos, desarrollar el coeficiente de correlación de Pearson con todas las variables de estudio y más tarde llevar a cabo el análisis de regresión múltiple. 


\section{Resultados}

La Tabla 1 presenta una síntesis de la información sociodemográfica de la muestra, donde la mayor proporción de encuestados son de sexo masculino (70.8\%), raza mestiza $(72.9 \%)$, solteros $(47.9 \%)$ o en unión libre $(41.7 \%)$, y nivel educativo inferior al bachillerato $(47.9 \%)$.

Tabla 1: Distribución muestral de proporciones de las variables sociodemográficas de los participantes $(n=48)$.

\begin{tabular}{llrr}
\hline \multicolumn{1}{c}{ Variable } & \multicolumn{1}{c}{ Valores } & N & $\%$ \\
\hline Sexo & Femenino & 14 & $29.2 \%$ \\
Estado Civil & Masculino & 34 & $70.8 \%$ \\
& Casado & 1 & $2.1 \%$ \\
& Separado & 3 & $6.3 \%$ \\
& Soltero & 23 & $47.9 \%$ \\
& Unión Libre & 20 & $41.7 \%$ \\
& Viudo & 1 & $2.1 \%$ \\
Nivel Educativo & Bachillerato & 9 & $18.8 \%$ \\
& Menos del bachillerato & 23 & $47.9 \%$ \\
& Ninguna & 11 & $22.9 \%$ \\
& No sabe & 5 & $10.4 \%$ \\
Ingreso Mensual & Menos de un salario mínimo & 6 & $12.5 \%$ \\
& No trabaja & 42 & $87.5 \%$ \\
\hline
\end{tabular}

La Tabla 2 presenta una síntesis de la prevalencia de diferentes factores determinantes de la salud entre los participantes. Es posible concluir que la mayor proporción de participantes no sufrió algún evento traumático (discapacidad, lesiones, muerte de seres queridos, amenazas, tortura, abuso sexual, víctima de violencia, etc.). 
Tabla 2: Distribución muestral de proporciones de distintos factores determinantes de la salud entre los participantes $(n=48)$.

\begin{tabular}{lcc}
\hline \multicolumn{1}{c}{ Variable } & $n$ & $\%$ \\
\hline Se Encuentra laborando & 20 & $41.7 \%$ \\
Sufre alguna discapacidad & 6 & $12.5 \%$ \\
Lesión física & 5 & $10.4 \%$ \\
Accidente laboral & 1 & $2.1 \%$ \\
Procedimientos médicos invasivos & 1 & $2.1 \%$ \\
Lesión o enfermedad & 9 & $18.7 \%$ \\
Accidente de vehículo motorizado & 2 & $4.2 \%$ \\
Perdida de la propiedad & 9 & $18.7 \%$ \\
Muerte de un ser querido & 24 & $50 \%$ \\
Lesión o enfermedad de un ser que rido & 10 & $20.8 \%$ \\
Amenaza a un ser querido & 10 & $20.8 \%$ \\
Testigo de violencia & 8 & $16.7 \%$ \\
Suicidio de un ser querido & 1 & $2.1 \%$ \\
Asalto sexual & 1 & $2.1 \%$ \\
Asalto físico & 2 & $2.1 \%$ \\
Violación & 1 & $2.1 \%$ \\
Robo & 5 & $2.1 \%$ \\
Amenaza de violencia física & 1 & $6.2 \%$ \\
Tortura & 1 & $2.1 \%$ \\
Víctima del crimen & 3 & $10.4 \%$ \\
Víctima de la violencia & 1 & $2.7 \%$ \\
Presenció un evento traumático & 1 & $2 \%$ \\
\hline & & 2 \\
\hline
\end{tabular}

La Tabla 3 presenta las medidas de tendencia central, de dispersión y alfa de Cronbach para las escalas $\mathrm{PCL}, \mathrm{K} 10, \mathrm{CBI}, \mathrm{HHI}$ y $\mathrm{WBC}$. Las escalas $\mathrm{PCL}, \mathrm{K} 10, \mathrm{CBI}$ y $\mathrm{HHI}$ presentan una alta fiabilidad, con valores alfa de Cronbach de $.87, .87, .93$ y .78 respectivamente. 
S. Vinaccia \& A. Ortega

Tabla 3: Medidas de tendencia central, de dispersión y alpha de Cronbach para las escalas PCL, K10, CBI, HHI y WBC.

\begin{tabular}{|c|c|c|c|c|c|c|c|c|c|c|}
\hline & $\mathrm{PCL}$ & $\mathrm{K} 10$ & $\mathrm{CBI}$ & $\mathrm{HHI}$ & $\begin{array}{l}\text { HHI- } \\
\text { Factor } \\
\text { I }\end{array}$ & $\begin{array}{l}\text { HHI- } \\
\text { Factor } \\
\|\end{array}$ & $\begin{array}{l}\mathrm{HHI}- \\
\text { Factor } \\
\text { III }\end{array}$ & WBC I & $\begin{array}{c}\text { WBC } \\
\|\end{array}$ & $\begin{array}{c}\text { WBC } \\
\text { III }\end{array}$ \\
\hline Mínimo & 0 & 9 & 0 & 12 & 6 & 6 & 4 & 1 & 1 & 1 \\
\hline Máximo & 41 & 50 & 38 & 48 & 16 & 16 & 33 & 10 & 3 & 4 \\
\hline Media & 10.38 & 14.54 & 16.00 & 39.73 & 12.92 & 13.75 & 14.04 & 7 & 1 & 1 \\
\hline Mediana & 8.00 & 13.00 & 15.46 & 41.00 & 13.00 & 14.00 & 13.00 & 6.28 & 1.31 & 1.73 \\
\hline DS & 9.11 & 6.73 & 12.54 & 7.15 & 2.35 & 2.26 & 3.63 & 3.38 & 0.624 & 1.026 \\
\hline Alpha & .871 & .873 & .926 & .776 & .623 & .610 & .721 & * & * & * \\
\hline
\end{tabular}

La Tabla 4 presenta las correlaciones bivariadas entre las escalas PCL, K10, CBI, HHI, y WBC. Las escalas $\mathrm{K} 10$ y $\mathrm{CBI}$ están correlacionadas significativamente con $\mathrm{PCL}(r=0.618$ y $r=0.414$ respectivamente), por tanto, las escalas $\mathrm{K} 10$ y $\mathrm{CBI}$ podrían ser predictores para $\mathrm{PCL}$; además se observó que los subdimensiones de esperanza que más se correlacionaron con esta fueron temporalidad y la prontitud con coeficientes de correlación de 0.58 y 0.82 , respectivamente.

Tabla 4: Matriz de correlaciones de las escalas y subescalas del estudio.

\begin{tabular}{lrrrrrrrrr}
\hline & 1 & 2 & 3 & 4 & 5 & 6 & 7 & 8 & 910 \\
\hline 1. PCL & - & & & & & & & & \\
2. K10 & $.62^{* *}$ & - & & & & & & & \\
3. CBI & $.41^{* *}$ & .23 & - & & & & & & \\
4. HHI & -.01 & .04 & .11 & - & & & & & \\
5. HHI-I & -.02 & .09 & -.08 & $.58^{* *}$ & - & & & & \\
6. HHI-II & .04 & .17 & .15 & $.82^{* *}$ & $.50^{* *}$ & - & & & \\
7. HHI-III & .05 & .14 & .18 & .02 & $.31^{*}$ & $.45^{* *}$ & - & & \\
8. WBC-I & -.19 & -.07 & $-.40^{* *}$ & .06 & .14 & .11 & -.05 & - & \\
9. WBC-II & -.10 & -.15 & .12 & .00 & -.16 & -.05 & -.01 & -.06 & - \\
10. WBC-III & -.12 & -.13 & -.11 & .02 & -.19 & .06 & -.07 & .033 & $.33^{*}-$ \\
\hline
\end{tabular}


De la tabla 4 observamos que las escalas K10 y CBI están correlacionadas significativamente con PCL $(r=.618$ y $r=.414$ respectivamente), por tanto, las escalas $\mathrm{K} 10$ y $\mathrm{CBI}$ podrían ser predictores para $\mathrm{PCL}$; además se observó que las dimensiones de esperanza que más se correlacionaron con esta fueron temporalidad y la prontitud con coeficientes de correlación de .578 y .817 respectivamente.

Con el fin de detectar posibles predictores del TEPT desarrolló un análisis de regresión múltiple por pasos, para predecir el TEPT presentado en la Tabla 5. Las variables entradas simultáneamente fueron: $\mathrm{K} 10, \mathrm{CBI}, \mathrm{HHI}, \mathrm{HHI}$-Factor I, HHI-Factor II, HHI-Factor III, WBC I, WBC II y WBC III. Se encontró que el modelo ajustado explica aproximadamente el $48 \%$ de la varianza total, en el cual los mejores predictores del TEPT en esta muestra fueron el $\mathrm{K} 10\left(B=.749^{* * *}\right)$ y $\mathrm{CBI}\left(\mathrm{B}=.222^{* *}\right)$.

Tabla 5: Análisis de regresión múltiple por pasos con estrés postraumático como variable criterio.

\begin{tabular}{lrrrrrrl}
\hline Variable & \multicolumn{1}{l}{ B } & \multicolumn{1}{l}{ Error tip. } & \multicolumn{1}{l}{$\beta$} & Sig. & $F$ & $g l$ & $\mathrm{R}^{2}$ \\
\hline Modelo & & & & & $3.71^{* * *}$ & 9.37 & .475 \\
K10 & .749 & .175 & .557 & $<.001$ & & & \\
CBI & .222 & .102 & .309 & .037 & & & \\
HHI & .010 & .479 & .008 & .983 & & & \\
HHI-I & .001 & .709 & .000 & .999 & & & \\
HHI-II & -.420 & 1.509 & -.103 & .783 & & & \\
HHI-III & -.130 & .572 & -.052 & .822 & & \\
WBC I & -.055 & .366 & -.021 & .881 & & & \\
WBC II & -.939 & 1.932 & -.065 & .630 & & & \\
WBC III & .029 & 1.192 & .003 & .981 & & & \\
\hline
\end{tabular}

\section{Discusión}

En la presente investigación participaron 48 sujetos guerrilleros desmovilizados pertenecientes al Frente 58 de las FARC-EP. Los resultados indicaron que los participantes al estudio no presentaron niveles significativos de estrés post traumático, tenían bajos niveles de estrés y de cambios en las creencias básicas. Por otra parte, presentaban niveles altos de esperanza y de bienestar físico y psicológico.

Estos resultados discrepan de los resultados reportados por diversos estudios epidemiológicos en excombatientes de las guerras de Irak y Afganistán, los que encuentran tasas de estrés 


\section{S. Vinaccia \& A. Ortega}

post traumático entre el $13.5 \%$ y el $30 \%$. En estos estudios, la prevalencia del TEPT se encuentra relacionada con la duración de la misión y el rol del soldado en el conflicto (Shalev, et al., 2017; Hunter, 2019).

Con relación a estudios realizados en el contexto del conflicto armado colombiano, los resultados del presente estudio presentan puntos de contacto con la investigación desarrollada por Reyes y Gama (2016) en 31 ex miembros de la AUC en la Cárcel de Alta Seguridad de Itagüí. En este caso, los sucesos estresantes y traumáticos más significativos para los participantes fueron la Muerte de Familiar, o de otro integrante de la red familiar externo al 1 grado de consanguineidad, y la muerte de los padres, respectivamente. Los eventos estresantes relacionados con actividades delictivas no fueron asociados al TEPT.

Por otra parte, los resultados reportados en este estudio se diferencian del estudio de Baldovino (2014), que evaluó la prevalencia de TEPT y TAP en 192 desmovilizados (115 hombres y 77 mujeres), desmovilizados de la FARC-EP y el ELN. El estudio reportó que entre $37 \%$ al $68 \%$ de los excombatientes presentaron criterios para TEPT y el $12.1 \%$ estaba en riesgo de presentarlos y de la investigación de Aponte y Zapata (2018), donde un 31\%, es decir, 8,370 personas ex combatientes colombianos, presentaban síntomas de estrés postraumático

Estas diferencias podrían en parte explicadas en parte por los resultados del estudio de Weierstall et al. (2013), que investigaron una muestra de 252 ex combatientes colombianos de fuerzas paramilitares y guerrilleras. El estudio reporta que la mayoría de los guerrilleros que se desmovilizaron individualmente y estaban cansados de luchar, tanto una atracción por la violencia, como un aumento de los síntomas de trauma, debido a su antiguo compromiso con el comportamiento violento. En contraste, entre aquellos que fueron desmovilizados colectivamente, la agresión apetitiva se asoció con un riesgo reducido de TEPT. La influencia de la experiencia de combate en la prevalencia de TEPT difiere notablemente entre los combatientes que se desmovilizaron individualmente, y los que eran miembros de un grupo que se vio obligado a desmovilizarse.

Nandi et al. (2016), estudiaron la relación entre tipos de eventos traumáticos, los actos violentos auto comprometidos, la gravedad de los síntomas del TEPT, y la agresión apetitiva entre 392 excombatientes en Burundi. La agresión apetitiva no se correlacionó con la gravedad de los síntomas del TEPT en la muestra total. Después de excluir a los participantes más gravemente traumatizados, la agresión apetitiva se relacionó negativamente con la gravedad de los síntomas del TEPT. Los hallazgos de tal estudio confirmaron hallazgos anteriores sobre la relación entre la agresión apetitiva y el TEPT. Se demostró que la agresión apetitiva reduce la gravedad de los síntomas del TEPT, pero no es la mejor protección contra el TEPT. 
Otro aspecto que podría haber influenciado en estos resultados es que, durante los diálogos de paz (2012-2016), las FARC siempre desestimaron el tema del TEPT en sus combatientes por ser considerado un enfoque "importado" que poco tenía que ver con las dificultades de sus ex integrantes. Ejemplo de esto es que un alto dirigente FARC planteaba durante los diálogos de paz "Yo creo que, en la guerra, nosotros estábamos muy unidos y eso como que combatía mucho eso, ¿no? Yo no creo que nosotros sufrimos realmente lo que sufren esos soldados norte americanos que van a Irak y que uno ve en documentales, que se vuelven locos y agresivos. Yo no veo eso tanto en la gente nuestra" (Pinto-García, 2021 p.5)

Por otro lado, el bajo nivel de estrés encontrado en la muestra de excombatientes de las FARC-EP discrepa con lo reportado en el Estudio Nacional de Salud Mental (Ministerio de Salud y Protección Social, 2015) en Colombia, el cual indica que el $40 \%$ de la población activa laboralmente, es decir entre 18 y 65 años, ha sufrido un trastorno psiquiátrico asociado al estrés que afecta el bienestar de las personas. Esto podría estar asociado a los pobres cambios observados a nivel de creencias centrales.

Las creencias centrales incluyen cómo creemos que se comportará la gente, cómo se desarrollarán los eventos y nuestra capacidad para influir en los eventos. Estas suposiciones dan estructura a los eventos en el mundo de un individuo, permiten que cada individuo planifique, prediga, y contribuya a cómo las personas y los eventos en el mundo son percibidos y comprendidos. Los investigadores han teorizado que "la ausencia de trauma es la desintegración abrupta del mundo interior" (Janoff-Bulman, 1992, p. 63).

Esto último podría estar relacionado, como lo plantea Ardila (2017), con que los guerrilleros de la FARC-EP, al haber llegado por lo general a sus filas de ambientes campesinos en etapas tempranas de la adolescencia con un alto déficit en habilidades básicas, terminan creciendo en esta organización militar con bajos niveles de autonomía y una limitada capacidad tomar decisiones propias y una relación con el mundo exterior muy limitada.

Los niveles altos de esperanza y bienestar prevalente asociados al proceso de paz y al proceso de reincorporación a la vida civil se relacionan con el modelo de Hert et al. (1991), "basándose en la percepción de que el resultado deseado es realista y probable y sentimiento de confianza con la iniciación de los planes para alcanzar las metas deseadas" (Farran, et al., 1995, p.62). Tener esperanza tiene consecuencias positivas en el individuo, como la posibilidad de sentir emociones positivas, entrenar el pensamiento para elaborar diferentes estrategias, ganar en flexibilidad y conseguir más simpatía y apoyo social, porque una actitud positiva es más bienvenida. La esperanza ha demostrado ser un fuerte mediador entre los efectos negativos del estrés y la satisfacción con la vida, disminuyendo los efectos negativos que el estrés tiene sobre ésta (Snyder, 2002). 
El análisis de regresión múltiple comprobó parcialmente la hipótesis de investigación al encontrarse que los niveles de distrés son predictores de estrés post traumático. Al respecto, la naturaleza de la conexión entre el distrés y el estrés post traumático no implica que todos los casos de trastorno de estrés agudo se conviertan finalmente en trastorno de estrés postraumático, esto es que no necesariamente todos los casos de estrés post traumático siguen a un distrés psicológico. Pese a esto, es común que los dos trastornos ocurran en secuencia después de una incidencia de trauma (Moore \& Penk, 2019).

Como limitación está por un lado el pequeño tamaño de la muestra de las FARC-EP encuestada, hecho que imposibilita generalizar estos resultados a la totalidad del Frente 58 y por otro que la evaluación psicológica que se desarrolló en "caliente" en su propio campamento en la selva del departamento de Córdoba, pocos días después de haber depuesto las armas. Por otro lado, se recomienda la validación y el desarrollo de instrumentos clínicos que aseguren la exactitud de las mediciones con esta población, considerando sus características socioculturales y académicas.

Se debe considerar la necesidad de desarrollar estudios que profundicen en las características del estado psicológico de los ex guerrilleros de la FARC-EP, con el fin de aclarar las variables involucradas que favorecen los procesos de reintegración a la vida civil, considerando que, tal como lo plantea Idrobo: "para que Colombia avance hacia una era de paz duradera y reconciliación, es de suma importancia que las víctimas y los excombatientes tengan un acceso adecuado a servicios de tratamiento de salud mental basados en la evidencia y a intervenciones para las condiciones psicológicas relacionadas con la guerra" (Idrobo et al 2018.p.268).

\section{Referencias}

Aguilera, T. (2017). Tomas y ataques guerrilleros (1965-2013). Centro Nacional de Memoria Histórica.

Amar, J., Abello, R., Madariaga, C., \& Ávila-Toscano, J. (2011). Relación entre redes personales y calidad de vida en individuos desmovilizados del conflicto armado colombiano. Universitas psychologica, 10(2), 355-369. https://doi.org/10.11144/Javeriana.upsy102.rrpc

Alexandre, N.M.C., \& Guirardello, E.B. (2002). Adaptación cultural de instrumentos utilizados en salud ocupacional. Revista Panamericana de Salud Pública, 11(2), 109-111. https://tinyurl.com/yckuv8bs

Aponte, D y Zapata. (2018). Salud mental en el contexto de DDR. Trayectorias, espectros de sufrimiento y calidad de vida en población desmovilizada del conflicto interno colombiano en proceso de reintegración. Universidad Externado de Colombia. 
Ardila, R. (2017). El mundo psicológico de los guerrilleros. Innovación y ciencia, 24(1), 11-19. https://tinyurl.com/yckth7ar

Baldovino, M. (2014). Prevalencia de estrés postraumático y trastorno antisocial de la personapersonalidad en la población desmovilizada de las guerrillas colombianas en Bogotá, D.C (Tesis de maestría). Universidad Católica de Colombia, Bogotá D.C., Colombia. https://tinyurl.com/bdynjdxp

Cann, A., Calhoun, L. G., Tedeschi, R. G., Kilmer, R. P., Gil-Rivas, V., Vishnevsky, T., \& Danhauer, S. C. (2010). The Core Beliefs Inventory: A brief measure of disruption in the assumptive world. Anxiety, stress \& coping, 23(1), 19-34. https://doi.org/dnv639

Centro Nacional de Memoria Histórica. (2015). Una nación desplazada. Informe nacional del desplazamiento forzado en Colombia. Centro Nacional de Memoria Histórica.

De la Espriella, R., Pingel, E. S. \& Falla, J. (2010). The (de)construction of a psychiatric diagnosis: PTSD among former guerrilla and paramilitary soldiers in Colombia. Global public health, 5(3), 221-232. https://doi.org/10.1080/17441691003709430

Estrada, J. (2013). La solución política y el proceso de paz en Colombia. Ocean Sur.

Farran, C. J., Herth, K. A., \& Popovich, J. M. (1995). Hope and hopelessness: critical clinical constructs. Sage.

Greenaway, K. H., Haslam, S. A., Cruwys, T., Branscombe, N. R., Ysseldyk, R., \& Heldreth, C. (2015). From "we" to "me": Group identification enhances perceived personal control with consequences for health and well-being. Journal of Personality and social psychology, 109(1), 53-74. https://doi.org/10.1037/pspi0000019

Hernández, R., Sampieri, R., Fernández Collado, C., \& Baptista, P. (2014). Metodología de la investigación (6a ed.). McGraw-Hill.

Herth, R. N. (1991). Development and refinement of an instrument to measure hope. Research and theory for nursing practice, 5(1), 39-51. https://tinyurl.com/4d6njuf7

Hunter, S. R. (2019). Drawing soldiers out of post-traumatic stress. Military medical research, 6, 5. https://doi.org/10.1186/s40779-019-0195-8

Idrobo F, Hessel P, Harker A, Evans-Lacko S, Avendaño M. (2018). Mental health of victims and exFARC members: A challenge for the peace process in Colombia. Lancet psychiatry, 5(6), 467-468. https://doi.org/10.1016/S2215-0366(18)30134-2 


\section{S. Vinaccia \& A. Ortega}

Janoff-Bulman, R. (1992). Shattered assumptions: Towards a new psychology of trauma. Free Press.

Kessler, R. C., Andrews, G., Colpe, L. J., Hiripi, E., Mroczek, D. K., Normand, S. L., Walters, E. E., \& Zaslavsky, A. M. (2002). Short screening scales to monitor population prevalences and trends in non-specific psychological distréss. Psychology medicine, 32(6), 959-976. https://doi.org/10.1017/s0033291702006074

Koebach, A., Carleial, S., Elbert, T., Schmitt, S., \& Robjant, K. (2021). Treating trauma and aggression with narrative exposure therapy in former child and adult soldiers: $A$ randomized controlled trial in Eastern DR Congo. Journal of consulting and clinical psychology, 89(3), 143-155. https://doi.org/10.1037/ccp0000632

Medina, C. (2017). Mafia, narcotráfico y bandas criminales en Colombia. Universidad Nacional.

Ministerio de Salud y Protección Social. (2015). Encuesta Nacional de Salud Mental (Vol. 1). Javegraf.

Moore, B., \& Penk, W. (2019). Treating PTSD in military personnel: a clinical handbook (2a ed.). Guilford Press.

Nandi, C., Crombach, A., Elbert, T., Bambonye, M., Pryss, R., Schobel, J., \& Weierstall-Pust, R. (2020). The cycle of violence as a function of PTSD and appetitive aggression: $A$ longitudinal study with Burundian soldiers. Aggressive behavior, 46(5), 391-399. https://doi.org/10.1002/ab.21895

Nandi, C., Crombach, A., Bambonye, M., Elbert, T., \& Weierstall, R. (2016). Appetitive aggression and its relation to posttraumatic stress in Burundian ex-combatants. Peace and conflict: Journal of peace psychology, 22(2), 102-108. https://doi.org/g7z2

Nandi, C., Crombach, A., Bambonye, M.,Elbert., \& Weierstall R. (2015) Predictors of posttraumatic stress and appetitive aggression in active soldiers and former combatants. European journal of psychotraumatology, 21(6), 26553. https://doi.org/f6884h

Pineda, J., Restrepo, M., Buenaventura, O \& Amariles, L. J. (2017). El proceso de inclusión laboral de los desmovilizados en la ciudad de Medellín y su área metropolitana. Revista logos, ciencia \& tecnología, $9(2), 10-26$. https://doi.org/10.22335/rlct.v9i2.477

Pinto-García, L. (2021, Abril 1). Resisting psychiatric temporalities in "Post-conflict" Colombia. Anthropology news. https://tinyurl.com/bdhjs5m2

Restrepo, E., \& Gagley, B. (2011). La desmovilización de los para militares en Colombia. Uniandes 
Reyes, N., \& Gama, A. (2016). Análisis correlacional entre el estrés post traumático, resiliencia, bienestar psicológico y personalidad en ex combatientes de la AUC en Bogotá D. C. (Trabajo de grado). Unisanitas, Bogotá, Colombia.

Ríos, J. (2016). Breve historia del conflicto armado en Colombia. EAN.

Shalev, A., Liberzon, I., \& Marmar, C. (2017). Post-traumatic stress disorder. New England jourjournal of medicine, 376(25), 2459-2469. https://doi.org/10.1056/nejmral612499

Snyder, C. R. (2002). Hope theory: Rainbows in the mind. Psychological inquiry, 13(4), 249-275. https://doi.org/10.1207/S15327965PLI1304_01

Tobón, C., Aguirre-Acevedo, D. C., Velilla, L., Duque, J., Ramos, C. P., \& Pineda, D. (2016). Perfil psiquiátrico, cognitivo y de reconocimiento de características emocionales de un grupo de excombatientes de los grupos armados ilegales en Colombia. Revista colombiana de psiquiatría, 45(1), 28-36. https://doi.org/10.1016/j.rcp.2015.07.004

Tobón, C., Ibañez, A., Velilla, L., Duque, J., Ochoa, J., Trujillo, N., Decety, J., \& Pineda, D. (2014). Emotional processing in Colombian ex-combatants and its relationship with empathy and executive functions. Social neuroscience, 10(2), 153-165. https://doi.org/g7z8

Ugarizza, J. \& Pabón, N. (2017). Militares y guerrillas. La memoria histórica del conflicto colombiano desde los archivos militares (1958-2016). Universidad del Rosario.

Valencia, O., \& Daza, M. F. (2011). Vinculación de grupos armados: un resultado del conflicto armado en Colombia. Diversitas, 6(2),429-239. https://doi.org/ghmmbw

Weathers, F. W., Litz, B. T., Keane, T. M., Palmieri, P. A., Marx, B. P., \& Schnurr, P. P. (2013). The PTSD checklist for DSM-5 (PCl-5). https://www.ptsd.va.gov

Weierstall, R., Castellanos, C. P. B., Neuner, F., \& Elbert, T. (2013). Relations among appetitive aggression, post-traumatic stress and motives for demobilization: a study in former Colombian combatants. Conflict and health, 7(1), 9. https://doi.org/gm29wg

\section{Para citar en APA}

Vinaccia, S. \& Ortega, A. (2021). Variables psicológicas asociadas al estrés Postraumático en desmovilizados de las Fuerzas Armadas Revolucionarias de Colombia (FARC-EP). Terapia Psicológica (En línea), 39(2), 257-272. https://doi.org/10.4067/S0718-48082021000200257 